\title{
Thoracic Blastomycosis from Morocco
}

\author{
E-mail: lahmiti@gmail.com
}

Received February 27, 2009; Accepted March 26, 2009; Published March 31, 2009

We read with great interest the paper by Cheikh Rouhou et al., which was recently published online in TheScientificWorldJOURNAL[1]. In this article, the authors report a new case of pulmonary and vertebral blastomycosis from Tunisia. They found that the probable reservoir of Blastomyces dermatitidis is the soil. Blastomycosis is considered a rare and sporadic disease in Africa, unlike in some endemic regions in Canada or in the upper Midwest in the U.S.

In fact, few cases of the disease were reported in the African literature and only one case was reported in the Moroccan literature. In 2005, there was a case of thoracic blastomycosis. It was primarily suspected to be a pulmonary carcinoma, but was never histologically confirmed. All the biopsies performed showed inflamed material. The medical student taking care of the patient found out that his patient sells used clothes (he imports second-hand clothes from the U.S. and Canada, and resells them in his store). Therefore, our medical student suspected that the patient's symptoms could be due to an imported disease. The medical staff recommended performing a mycological study. Culture on Sabouraud's dextrose agar at $25^{\circ} \mathrm{C}$ further confirmed the presence of $B$. dermatitidis northamericanus. A mycological study was then performed on the clothes sold by the patient. It showed a considerable amount of blastomycosis spores, a finding that concludes that the infection was due to massive and chronic inhalation of the spores present in the imported clothes. The patient was treated with a daily 400-mg dose of fluconazole for 12 months, a treatment that led to a great clinical and radiological improvement. So far, the patient has shown no evidence of recurrence.

Our case illustrates two important facts: the first is that medical students play an important role in the diagnosis and treatment of new diseases in our university hospitals, and the second is that imported clothes are not always safe. There should be a strict and thorough verification of imported clothes in order to prevent deadly disease.

Saad Lahmiti, M.D.

Salwa Baki, M.D.

University Hospital Mohammed VI Marrakech

\section{REFERENCE}

1. Cheikh Rouhou, S., Racil, H., Ismail, O., Trabelsi, S., Zarrouk M., Chaouch, N., Hantous, S., Khaled, S., El Mezni, F., and Chabbou, A. (2008) Pulmonary blastomycosis: a case from Africa. TheScientificWorldJOURNAL 8, 10981103. DOI 10.1100/tsw.2008.141

This article should be cited as follows:

Lahmiti, S. and Baki, S. (2008) Thoracic blastomycosis from Morocco. TheScientificWorldJOURNAL 8, 200. DOI 10.1100/tsw.2008.30. 